\title{
SíNDROME DE DELEÇÃO 22q11.2: IMPORTÂNCIA DA AVALIAÇÃO CLÍNICA E TÉCNICA DE FISH
}

Dayane Bohn Koshiyama ${ }^{1}$, Rafael Fabiano Machado Rosa², Paulo Ricardo Gazzola Zen ${ }^{3}$, Vera lúcia Berenstein Pereira ${ }^{4}$, Carla Graziadio ${ }^{5}$, Virgínia Maria Cóser ${ }^{6}$, Cláudia Pires Ricachinevsky ${ }^{7}$, Marileila Varella-Garcia ${ }^{8}$, Giorgio Adriano Paskulin ${ }^{9 *}$

Trabalho realizado na Universidade Federal de Ciências da Saúde de Porto Alegre

*Correspondência:

Rua Sarmento Leite, $n^{\circ} 245 / 403$

Porto Alegre - RS

CEP: $90050-170$

Telefone: (51) 3303-8771

Fax: (51) 3303-8810

paskulin@ufcspa.edu.br

\begin{abstract}
RESUMO
ОвлEтIvo. A síndrome de deleção 22q11.2 é considerada hoje uma das doenças genéticas mais frequentes em humanos. Caracteriza-se clinicamente por um espectro fenotípico bastante amplo, com mais de 180 achados já descritos, tanto físicos como comportamentais. Contudo, nenhum deles é patognomônico ou mesmo obrigatório, o que acaba dificultando o diagnóstico. Assim, o objetivo do presente estudo foi determinar a prevalência e as características clínicas de pacientes com microdeleção 22q11.2 em uma amostra selecionada de indivíduos com suspeita clínica de síndrome de deleção 22q11.2 e cariótipo normal.

MÉTodos. Uma amostra selecionada de 30 pacientes com suspeita clínica da síndrome de deleção 22q11.2 e cariótipo normal foi avaliada através da aplicação de um protocolo clínico padrão e análise citogenética por meio da técnica de hibridização in situ fluorescente.

Resultados. A microdeleção 22q11.2 foi identificada em três pacientes (10\%), sendo esta prevalência similar a da maioria dos estudos descritos na literatura que oscila de $4 \%$ a $21 \%$. Os pacientes com síndrome de deleção 22q11.2 do nosso trabalho se caracterizaram por um fenótipo variável, com poucos achados clínicos similares, o que foi concordante com a descrição da literatura.

Conclusão. Nossos achados reforçam a ideia de que o diagnóstico clínico da síndrome de deleção 22q11.2 é difícil devido à sua grande variabilidade fenotípica. Assim, uma avaliação clínica detaIhada associada a um teste sensível como a hibridização in situ fluorescente, são fundamentais para a identificação destes pacientes.
\end{abstract}

Unitermos: Síndrome de Deleção 22q11.2. Síndrome de DiGeorge. FISH. Cromossomos Humanos Par 22.

\section{INTRODUÇão}

A síndrome de deleção 22q11.2 (SD22q11) (OMIM \#188400 / \#192430¹), identificada no começo da década de $90^{2}$, é considerada hoje uma das doenças genéticas mais freqüentes em humanos, com uma prevalência estimada de 1 para cada 2.000-6.000 nascimentos ${ }^{3,4,5}$. Considerando-se 0 quão comum e variável é a SD22q11, não é de se surpreender que esta síndrome tenha sido independentemente descrita em diferentes momentos e de diferentes formas, em várias partes do mundo, refletindo a visão de vários especialistas para a mesma doença. Em consequência dos vários nomes ficou a falsa impressão de que existem diferentes doenças associadas à deleção 22q11.2: síndrome de DiGeorge, síndrome velocardiofacial, síndrome de Sedlačková, síndrome de Cayler, síndrome de Shprintzen e CATCH22 (Conotruncal heart defect, Abnormal face, T-cell deficiency, Clefting, e Hypocalcemia, decorrentes de uma anormalidade no cromossomo 22) ${ }^{6}$. Contudo, todas essas doenças representam a mesma condição que tem uma expressão fenotípica altamente variável ${ }^{4,6}$.

Até o momento, mais de 180 achados clínicos já foram descritos na SD22q11, tanto do ponto vista físico como comportamental. No entanto, nenhum deles é patognomônico ou mesmo obrigatório, o que acaba dificultando o seu diagnóstico ${ }^{3,6}$. Estudos recentes têm mostrado que, em amostras de pacientes com suspeita clínica de SD22q11, a microdeleção 22q11.2 tem sido descrita, de forma geral, em $4 \%$ a $21 \%$ dos indivíduos ${ }^{7,8}$, sendo que trabalhos similares são raros em nosso meio ${ }^{9}$.

Assim, o objetivo do presente estudo foi determinar a prevalência e as características clínicas de pacientes com microdeleção 22q11.2, identificados através da técnica de hibridização in situ

1,4. Universidade Federal de Ciências da Saúde de Porto Alegre - UFCSPA, Porto Alegre, RS

2,3,5,9. Universidade Federal de Ciências da Saúde de Porto Alegre - UFCSPA e Complexo Hospitalar Santa Casa de Porto Alegre - CHSCPA, Porto Alegre, RS

6. Hospital Universitário de Santa Maria, Santa Maria, RS

7. Hospital da Criança Santo Antônio - HCSA/Complexo Hospitalar Santa Casa de Porto Alegre - CHSCPA, Porto Alegre, RS

8. University of Colorado Health Sciences Center, Aurora, Colorado, USA 
fluorescente (FISH), em uma amostra selecionada de indivíduos com suspeita clínica de SD22q11 e cariótipo por banda GTG normal.

\section{MÉTodos}

\section{Pacientes}

A amostra foi constituída por uma coorte selecionada de pacientes com suspeita clínica de SD22q11, avaliados por médicos especialistas da Genética Clínica da Universidade Federal de Ciências da Saúde de Porto Alegre (UFCSPA)/ Complexo Hospitalar Santa Casa de Porto Alegre (CHSCPA), Rio Grande do Sul, Brasil. Foram incluídos no estudo somente pacientes que apresentavam avaliação cariotípica por banda GTG normal e pelo menos dois achados clínicos sugestivos da SD22q11, como anormalidades de palato; malformações cardiovasculares (especialmente do tipo conotruncal); déficit de aprendizagem ou de fala; transtornos comportamentais; anormalidades oftalmológicas; alterações imunológicas ou hipoplasia/ agenesia de timo, e hipocalcemia. Esta abordagem foi concordante com a adotada nos trabalhos de Tobias et al. (1999) ${ }^{10} \mathrm{e}$ Brunet et al. (2006) ${ }^{8}$. No exame físico dos pacientes, dismorfias maiores e menores foram anotadas seguindo a terminologia descrita por Merks et al. (2003) ${ }^{11}$. Medidas antropométricas (comprimento/altura, peso e perímetro cefálico) foram avaliadas através de curvas padrões de crescimento ${ }^{12}$. Valores dois-desvios padrão acima ou abaixo da média, com as devidas correções para o comprimento/altura, foram consideradas anormais. Anormalidades em outros órgãos ou sistemas, identificadas através de exames e avaliações adicionais solicitados devido a sintomas clínicos específicos de alguns pacientes, foram verificadas.

O presente estudo foi aprovado pelos Comitês de Ética em Pesquisa da UFCSPA e do CHSCPA. Somente foram incluídos pacientes cujos pais consentiram em participar do trabalho.

\section{Cariótipo de alta resolução}

O cariótipo de alta resolução ( $\geq 550$ bandas) foi realizado no Laboratório de Citogenética da UFCSPA através da técnica modificada de Yunis (1981) ${ }^{13}$. Resumidamente, esta inclui um cultivo celular por 72 horas de linfócitos estimulados com fitohemaglutinina, sincronização com o uso de metotrexato/timidina e coloração por bandamento GTG. A análise das lâminas de cada caso foi realizada em microscópio Axioskop Zeiss através da avaliação de 25 placas metafásicas.

\section{Técnica de FISH para microdeleção 22q11.2}

As lâminas para análise pela técnica de FISH foram preparadas a partir do material fixado (suspensão de células do cultivo sincronizado estocado em solução de Carnoy a $-20^{\circ} \mathrm{C}$ ). A sonda de DNA comercialmente disponível DiGeorge/VCFS Region (TUPLE1) (Abbott Laboratories, Des Planes, Illinois, EUA) foi utilizada nos experimentos, seguindo um protocolo padrão de codesnaturação ${ }^{14}$. Em cada caso, foram analisados 100 núcleos interfásicos e 20 placas metafásicas por meio de microscópio de epifluorescência Olympus BX51, equipado com filtros Texas Red, FITC, DAPI e triplo. Núcleos interfásicos rompidos, sobrepostos ou com um importante sinal de fundo, bem como placas
Figura 1 - Aparência craniofacial, frente (A) e perfil (B), de um dos pacientes com microdeleção 22q11.2

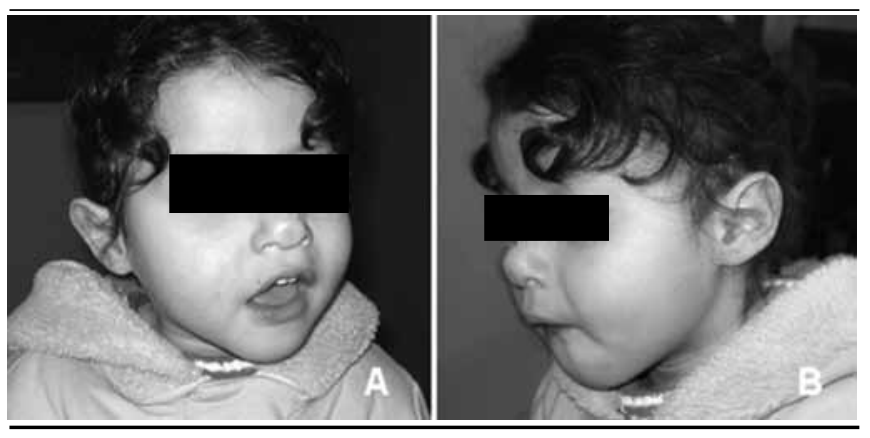

metafásicas com alto sinal de fundo, incompletas, ou muito próximas entre si, foram excluídos da análise. Os resultados foram interpretados de acordo com as normas presentes no International System for Human Cytogenetic Nomenclature (ISCN) $2005^{15}$.

\section{Análise estatística}

A análise dos dados foi realizada através do programa PEPI (versão 4.0). O teste estatístico utilizado foi o teste exato de Fisher bicaudado para comparação de frequências. Foram considerados como significantes valores de $\mathrm{P}<0,05$.

\section{Resultados}

A amostra final foi composta por 30 pacientes que preencheram os critérios de inclusão propostos e cujos pais consentiram em participar do estudo. Destes, 18 (60\%) eram do sexo masculino e 12 (40\%) do feminino. Suas idades variaram de um dia de vida até 16 anos, sendo que cerca de metade deles (53\%) encontrava-se no primeiro ano de vida. A análise através da técnica de FISH foi realizada com sucesso em todos os casos, sendo que a microdeleção 22q11.2 foi verificada em três pacientes (10\%). A Figura 1 e a Tabela 1 expõem as características clínicas apresentadas pelos mesmos.

\section{Discussão}

Apesar dos estudos citogenéticos através do cariótipo de alta resolução serem capazes de identificar uma pequena parcela de pacientes com a SD22q11 (15\% ou menos) ${ }^{16}$, a maioria destes indivíduos apresenta deleções menores (microdeleções) detectadas somente pela técnica de $\mathrm{FISH}^{3,17}$. Esta é uma técnica que integra a utilização da citogenética clássica com a genética molecular, através do uso de sondas de DNA marcadas com material fluorescente que identificam regiões específicas do genoma $^{18}$. A sonda DiGeorge/VCFS Region (TUPLE 1) utilizada no presente estudo é uma combinação de dois reagentes, a sonda SpectrumOrange ${ }^{T M}$ LSI TUPLE 1 (HIRA) (que reconhece o gene TUPLE 1 e os microssatélites D22S553, D22S609, e D22S942, localizados dentro da região comumente deletada na síndrome de deleção 22q11.2) e a sonda de controle SpectrumGreen ${ }^{T M}$ LSI ARSA (homóloga ao gene da arilsulfatase A, localizado na região 22q13.3). Os dois reagentes da sonda hibridizada, TUPLE 
Koshiyama DB et aL.

1 e ARSA, fluorescem com intensidade moderada a brilhante, sendo que em núcleos de células normais geralmente aparecem como dois pares de sinais distintos, um vermelho e um verde. Por sua vez, em uma metáfase normal, a sonda tipicamente se apresenta como pequenos sinais, um em cada cromátide de cada cópia do cromossomo 22 (Figura 2).

A frequência de microdeleção 22q11.2 verificada em nosso estudo (10\%) foi semelhante à descrita na maioria dos trabalhos da literatura que também avaliaram pacientes com suspeita clínica da SD22q11 através da técnica de FISH, variando de $4 \%$ a $21 \%(P>0,05)^{7,8,19,20,21,22}$. Diferença estatisticamente significante $(P<0,001)$ foi encontrada somente em relação ao trabalho de Sandrin-Garcia et al. (2007) ${ }^{9}$, único estudo similar realizado no Brasil, que avaliou 16 pacientes com suspeita da SD22q11 e detectou uma frequência de deleção de $62 \%$. Esta diferença pode ser explicada, pelo menos em parte, pela forma de seleção da amostra analisada e pela metodologia empregada no estudo. No trabalho de Sandrin-Garcia et al. (2007) os autores avaliaram pacientes com suspeita clínica de SD22q11 sem cardiopatia congênita e com idade superior a seis anos (a média foi de 14 anos $)^{9}$, período em que muitas das características clínicas da síndrome, como insuficiência velofaríngea, déficit cognitivo e de linguagem, transtornos comportamentais e até mesmo o aspecto facial, tornam-se mais evidentes ${ }^{3,4}$.

É sabido que existem alguns achados clínicos, como fenda palatina, sequência de Pierre-Robin e defeitos cardíacos conotruncais, que deveriam levar a um alto índice de suspeição da SD22q11 $1^{4,5,6}$. Além disso, cerca de $50 \%$ a $75 \%$ dos pacientes

Tabela 1 - Características clínicas apresentadas pelos pacientes da amostra, divididos de acordo com 0 resultado da pesquisa de microdeleção $22 q 11.2$ pela técnica de FISH

\begin{tabular}{|c|c|c|c|}
\hline $\begin{array}{l}\text { Pacientes } \\
\text { Achados clínicos }\end{array}$ & $\begin{array}{c}\text { Microdeleção } \\
22 q 11.2\end{array}$ & $\begin{array}{c}\text { FISH } \\
\text { Normais }\end{array}$ & Total \\
\hline Idade & $23 d-12 a$ & $1 d-16 a 6 m$ & $1 d-16 a 6 m$ \\
\hline Sexo & $1 \mathrm{M} / 2 \mathrm{~F}$ & $17 \mathrm{M} / 10 \mathrm{~F}$ & $18 \mathrm{M} / 12 \mathrm{~F}$ \\
\hline Retardo de crescimento & $1 / 3$ & $6 / 27$ & $7 / 30$ \\
\hline $\mathrm{RDNPM}^{*}$ & $2 / 2$ & $10 / 18$ & $12 / 20$ \\
\hline Atraso na fala* & $2 / 2$ & $5 / 18$ & $7 / 20$ \\
\hline Transtorno comportamental* & $1 / 1$ & - & $1 / 11$ \\
\hline Plagiocefalia & - & $1 / 27$ & $1 / 30$ \\
\hline Macrocefalia & - & $1 / 27$ & $1 / 30$ \\
\hline Achados faciais dismórficos & $2 / 3$ & $24 / 27$ & $26 / 30$ \\
\hline Paralisia facial & - & $1 / 27$ & $1 / 30$ \\
\hline Estrabismo & $1 / 3$ & $1 / 27$ & $2 / 30$ \\
\hline Coloboma de íris & - & $1 / 27$ & $1 / 30$ \\
\hline Fosseta pré-auricular & - & $2 / 27$ & $2 / 30$ \\
\hline Atresia de coanas & - & $1 / 27$ & $1 / 30$ \\
\hline Fenda palatina & - & $6 / 27$ & $6 / 30$ \\
\hline Apêndice branquial & - & $1 / 27$ & $1 / 30$ \\
\hline Laringo/traqueomalácia & - & $3 / 27$ & $3 / 30$ \\
\hline Membrana anterior laríngea & $1 / 3$ & - & $1 / 30$ \\
\hline Agenesia de timo & $1 / 3$ & - & $1 / 30$ \\
\hline Atresia de esôfago & - & $1 / 27$ & $1 / 30$ \\
\hline Cardiopatia congênita & $3 / 3$ & $22 / 27$ & $25 / 30$ \\
\hline Conotruncal & $2 / 3$ & $13 / 27$ & $15 / 30$ \\
\hline Sequência de Poland & - & $1 / 27$ & $1 / 30$ \\
\hline Escoliose & - & $2 / 27$ & $2 / 30$ \\
\hline Dedos das mãos afilados & $1 / 3$ & $4 / 27$ & $5 / 30$ \\
\hline Camptodactilia & - & $1 / 27$ & $1 / 30$ \\
\hline Polegares baixo implantados & - & $1 / 27$ & $1 / 30$ \\
\hline Baço acessório & $1 / 3$ & $1 / 27$ & $2 / 30$ \\
\hline Anormalidades renais & $2 / 3$ & $3 / 27$ & $5 / 30$ \\
\hline Hipospádia* & - & $1 / 17$ & $1 / 18$ \\
\hline Criptorquidia* & $1 / 1$ & $4 / 17$ & $5 / 18$ \\
\hline Luxação congênita de quadril & - & $1 / 27$ & $1 / 30$ \\
\hline Sobreposição dos dedos dos pés & - & $3 / 27$ & $3 / 30$ \\
\hline Pé torto congênito & - & $1 / 27$ & $1 / 30$ \\
\hline Hipocalcemia / Hipoparatireoidismo & $1 / 3$ & $6 / 27$ & $7 / 30$ \\
\hline Trombocitopenia & $1 / 3$ & $3 / 27$ & $4 / 30$ \\
\hline
\end{tabular}

d, dias; m, meses; a, anos; M, masculino; F, feminino; RDNPM, retardo do desenvolvimento neuropsicomotor.

* Achados avaliados em função da idade e do sexo. 


\section{Figura 2- Placas metafásicas pela técnica de FISH mostrando, respectivamente, um padrão normal de hibridização, com presença dos sinais esperados em ambos os cromossomos 22 (A) e ausência do sinal correspondente à região TUPLE 1 (sinal vermelho) em um dos cromossomos 22, compatível com microdeleção 22q11.2 (B). As setas brancas indicam a localização dos cromossomos 22 nas duas placas metafásicas}

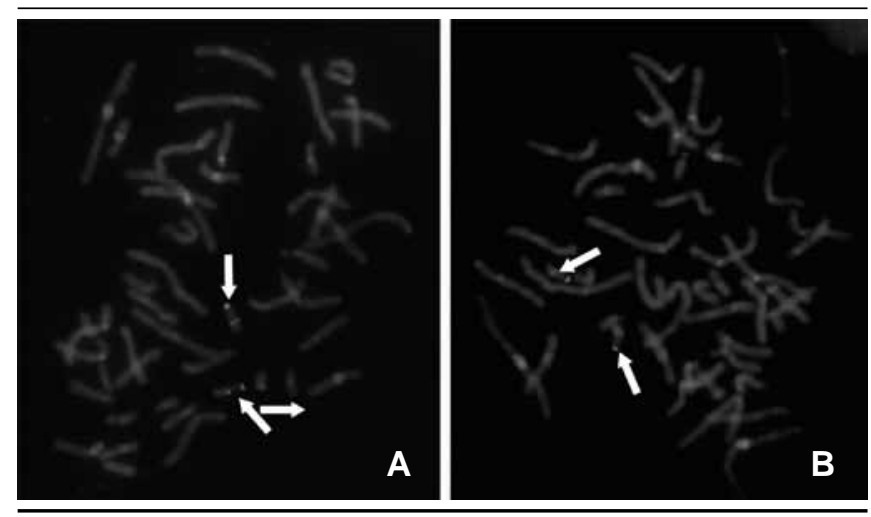

com a síndrome apresentariam sinais precoces de uma malformação congênita maior que poderiam auxiliar na realização do seu diagnóstico ainda durante a infância ${ }^{23}$. Contudo, a grande variabilidade clínica observada na SD22q11, mesmo intrafamiliar, tem dificultado em muito o seu reconhecimento ${ }^{3,24}$, sendo observadas diferenças mesmo entre gêmeos monozigóticos ${ }^{25}$. Apesar da similaridade de alguns achados clínicos, nossos pacientes com SD22q11 se caracterizaram por um fenótipo variável, o que foi concordante com a descrição feita na literatura. De forma interessante, nenhum dos pacientes com fenda palatina apresentou a deleção, sendo que defeitos cardíacos conotruncais foram frequentes mesmo no grupo sem a síndrome. Por outro lado, alguns achados, como agenesia de timo, membrana anterior laríngea, interrupção do arco aórtico e transtorno comportamental, foram observados somente em pacientes com a SD22q11; contudo, é sabido que os mesmos não são patognomônicos ou obrigatórios para a síndrome ${ }^{4}$, como pode ser observado pela própria Tabela 1 .

Devemos nos lembrar também que em aproximadamente $2 \%$ a $3 \%$ dos casos os pacientes podem apresentar rearranjos ou deleções ainda menores em 22q11.2, identificados somente através de técnicas moleculares diferentes do FISH, como a reação em cadeia da polimerase (PCR) e o sequenciamento ${ }^{26,27}$. Independentemente de tudo isso, é importante notar que indivíduos com a SD22q11, devido ao grande número de anormalidades muitas vezes associadas, são considerados pacientes em risco, pois frequentemente necessitam de uma avaliação e manejo multidisciplinar, envolvendo especialidades como a Pediatria, Genética Médica, Cardiologia, Otorrinolaringologia, Cirurgia Plástica, Imunologia, Endocrinologia e Psiquiatria 5,6,26,28.

\section{Conclusão}

Apesar da descrição de achados clínicos sugestivos para SD22q11, nossos resultados, em concordância com a literatura, reforçam a ideia de que o diagnóstico clínico da síndrome é difícil devido à sua grande variabilidade fenotípica. Assim, a realização de uma avaliação clínica detalhada associada a um teste sensível como o FISH, são fundamentais para a identificação destes pacientes. Infelizmente, o acesso a este exame em nosso meio é ainda bastante restrito, devido, principalmente, ao seu alto custo. Além disso, são raros os centros em nosso país que disponibilizam de infraestrutura básica necessária para a realização e desenvolvimento da técnica de FISH. Por outro lado, a análise inicial através do exame de cariótipo também é importante, pois outras anormalidades cromossômicas, como deleções do braço curto do cromossomo 10 e do braço longo do cromossomo 4 , podem simular um fenótipo sugestivo de SD22q113,4,29. Além disso, uma pequena percentagem $(<1 \%)$ de pacientes com a SD22q11 apresenta rearranjos cromossômicos, como translocações envolvendo a região q11 do cromossomo 22, detectados pelo cariótipo ${ }^{26}$.

A importância da confirmação do diagnóstico da SD22q11 reside no fato de que o mesmo proporciona tanto o adequado manejo clínico destes indivíduos como o correto aconseIhamento genético de suas famílias. A deleção segrega nas famílias com um padrão de herança autossômico dominante, ou seja, indivíduos portadores apresentam um risco de 50\% de transmitirem a deleção a seus filhos. Ambos os pais de um paciente com a SD22q11, mesmo na ausência de sinais sugestivos da síndrome, devem ser testados com o objetivo de excluir a possibilidade de que um deles apresente a deleção ${ }^{30}$. Esta avaliação é particularmente importante se os mesmos ou outros membros da família estão considerando a possibilidade de ter outros filhos. Todos estes fatores, associados à alta prevalência da SD22q11 e de outras doenças malformativas e oncológicas nas quais os pacientes se beneficiariam da realização de técnicas diagnósticas moleculares como a de FISH, justificariam a implementação em nosso país de mais centros capazes de realizar estes exames.

\section{Conflito de interesse: não há}

\section{SUMMARY}

22q11.2 DELETION SYNDROME: IMPORTANCE OF CLINICAL EVALUATION AND FISH ANALYSIS

OBJECTIVE. The 22q11.2 deletion syndrome nowadays is considered one of the most often observed genetic diseases in humans. It is clinically characterized by a rather wide phenotypic spectrum, with more than 180 clinical features physical as well as behavioral, already described. However, none is pathognomonic or obligatory which makes diagnosis even more difficult. Thus, this study intended to determine the prevalence and clinical characteristics of patients with 22q11.2 microdeletion in a selected sample of subjects with clinical suspicion of 22q11.2 deletion syndrome and normal karyotype.

Methods. A selected sample of 30 patients with clinical suspicion of 22q11.2 deletion syndrome and normal karyotype was evaluated by application of a standard clinical protocol and cytogenetic analysis with fluorescent in situ hybridization.

RESULTS. 22q11.2 microdeletion was identified in 3 patients (10\%), a prevalence similar to the majority of published studies, which ranged from 4 to $21 \%$. The $22 q 11.2$ deletion syndrome patients in this study were characterized by a variable phenotype 
KoshiYama DB et aL.

\section{and shared few clinical features, in agreement with the literature description.}

CONCLUSIONS. These findings strengthen the idea that clinical diagnosis of 22q11.2 deletion syndrome is difficult due to the large phenotypic variability. Therefore a detailed clinical evaluation associated to a sensitive test such as fluorescent in situ hybridization analysis is crucial for the identification of these patients. [Rev Assoc Med Bras 2009; 55(4): 442-6]

KEY WORDS: 22q11.2 Deletion Syndrome. DiGeorge Syndrome. FISH. Chromosomes Human pair 22.

\section{REFERÊNCIAS}

1. Online Mendelian Inheritance in Man. OMIM (TM). [cited 2008 jun 12]. Baltimore: BeMcKusick-Nathans Institute for Genetic Medicine, Johns Hopkins University. Bethesda :National Center for Biotechnology Information, National Library of Medicine; 2000. Avaliable from: http://www.ncbi.nlm.nih.gov/omim/.

2. Scambler PJ, Kelly D, Lindsay E, Williamson R, Goldberg R, Shprintzen R, Wilson DI, Goodship JA, Cross IE, Burn J. Velo-cardio-facial syndrome associated with chromosome 22 deletions encompassing the DiGeorge locus. Lancet. 1992;339:1138-9.

3. Thomas JA, Graham JM Jr. Chromosome 22q11 deletion syndrome: an update and review for the primary pediatrician. Clin Pediatr (Phila). 1997;36:253-66.

4. Shprintzen RJ. Velo-cardio-facial syndrome. In: Cassidy SB, Allanson JE, editors. Management of genetic syndromes. 2nd ed. New York: Wiley-Liss; 2005. p.615-31.

5. Kobrynski L, Sullivan KE. Velocardiofacial syndrome, DiGeorge syndrome: the chromosome 22q11.2 deletion syndromes. Lancet. 2007;370:1443-52.

6. Robin NH, Shprintzen RJ. Defining the clinical spectrum of deletion 22q11.2. J Pediatr. 2005;147:90-6.

7. Smith A, Heaps LS, Robson L. Apparently unrelated cytogenetic abnormalities among 462 probands referred for the detection of $\operatorname{del}(22 q)$ by FISH. Am J Med Genet. 2002;113:346-50.

8. Brunet A, Gabau E, Perich RM, Valdesoiro L, Brun C, Cabalin MR, Guitart M. Microdeletion and microduplication 22q11.2 screening in 295 patients with clinical features of DiGeorge/Velocardiofacial syndrome. Am J Med Genet. 2006; 140:2426-32.

9. Sandrin-Garcia P, Macedo C, Martelli LR, Ramos ES, Guion-Almeida ML, Richieri-Costa A, Passos GA. Recurrent 22q11.2 deletion in a sibship suggestive of parental germline mosaicism in velocardiofacial syndrome. Clin Genet. 2002;61:380-3.

10. Tobias ES, Morrison N, Whiteford ML, Tolmie JL. Towards earlier diagnosis of 22q11 deletions. Arch Dis Child. 1999;81:513-4.

11. Merks JH, van Karnebeek CD, Caron HN, Hennekam RC. Phenotypic abnormalities: terminology and classification. Am J Med Genet. 2003;123:211-30.

12. Jones KL. Smith's recognizable patterns of human malformation. 6th ed. Philadelphia: Elsevier Saunders; 2006.

13. Yunis, JJ. New chromosome techniques in the study of human neoplasia. Hum Pathol. 1981;12:540-9.

14. Helfrich BA, Raben D, Varella-Garcia M, Gustafson D, Chan DC, Bemis L, et al. Antitumor Activity of the Epidermal Growth Factor Receptor (EGFR) Tyrosine kinase inhibitor gefitinib (ZD1839, Iressa) in non-small cell lung cancer cell lines correlates with gene copy number and EGFR mutations but not EGFR protein levels. Clin Cancer Res. 2006;12:7117-25

15. Shaffer LG, Tommerup N, editores. ISCN. An international system for human cytogenetic nomenclature. Basel: S. Karger; 2005

16. Driscoll DA, Spinner NB, Budarf ML, McDonald-McGinn DM, Zackai EH, Goldberg RB et al. Deletions and microdeletions of 22q11.2 in velo-cardio-facial syndrome. Am J Med Genet. 1992;44:261-8.

17. Lindsay E, Goldberg R, Jurecic V, Morrow B, Carlson C, Kucherlapatti RS, et al. Velo-cardio-facial syndrome: frequency and extent of 22q11 deletions. Am J Med Genet. 1995;57:514-22.

18. Stumm M, Tönnies H, Wieacker PF. Molecular cytogenetic techniques for the diagnosis of chromosomal abnormalities in childhood disease. Eur J Pediatr. 1999;158:531-6.

19. Bartsch O, Nemecková M, Kocárek E, Wagner A, Puchmajerová A, Poppe M, et al. DiGeorge/Velocardiofacial syndrome: FISH studies of chromosomes 22q11 and 10p14, and clinical reports on the proximal 22q11 deletion. Am J Med Genet. 2003;117:1-5.

20. Cotter PD, Nguyen H, Tung G, Rauen KA. Incidence of microduplication $22 q 11.2$ in patients referred for FISH testing for velocardiofacial and Di George syndromes. Eur J Med Genet. 2005;13:1245-6.

21. Ensenauer RE, Adeyinka A, Flynn HC, Michels VV, Lindor NM, Dawson DB, et al. Microduplication 22q11.2, an emerging syndrome: clinical, cytogenetic, and molecular analysis of thirteen patients. Am J Hum Genet. 2003;73:1027-40.

22. Kitsiou-Tzeli S, Kolialexi A, Fryssira H, Galla-Voumvouraki A, Salavoura K, Kanariou M, et al. Detection of 22q11.2 deletion among 139 patients with DiGeorge/Velocardiofacial syndrome features. In Vivo. 2004;18:603-8.

23. Swillen A, Vogels A, Devriendt K, Fryns JP. Chromosome $22 q 11$ deletion syndrome: update and review of the clinical features, cognitive-behavioral spectrum, and psychiatric complications. Am J Med Genet. 2000:97:128-35.

24. Digilio MC, Angioni A, De Santis M, Lombardo A, Giannotti A, Dallapiccola B, et al. Spectrum of clinical variability in familial deletion 22q11.2: from full manifestation to extremely mild clinical anomalies. Clin Genet . 2003;63:308-13.

25. Vincent MC, Heitz F, Tricoire J, Bourroillou G, Kuhlein E, Rolland M et al. 22q11 deletion in DGS/VCFS monozygotic twins with discordant phenotypes. Genet Couns. 1999;10:43-9.

26. 22q11.2 Deletion Syndrome. Gene Clinics, Gene Reviews. [cited 2008 jun 16]. Seattle: University of Washington; 2003. Available from: http://www. geneclinics.org

27. Carlson C, Sirotkin H, Pandita R, Goldberg R, McKie J, Wadey R, et al. Molecular definition of 22q11 deletions in 151 velo-cardio-facial syndrome patients. Am J Hum Genet. 1997;61:620-9.

28. Greenhalgh KL, Aligianis IA, Bromilow G, Cox H, Hill C, Stait Y, et al. 22q11 deletion: a multisystem disorder requiring multidisciplinary input. Arch Dis Child. 2003;88:523-4.

29. Fernández L, Lapunzina P, Pajares IL, Palomares M, Martinez I, Fernández $B$, et al. Unrelated chromosomal anomalies found in patients with suspected 22q11.2 deletion. Am J Med Genet. 2008;146A:1134-41.

30. Smith A, Robson L. Low frequency of inherited deletions of 22q11. Am J Med Genet. $1999 ; 85: 513-4$ Aceito para publicação: 12/02/09 